\title{
A holey fiber based Brillouin laser
}

\author{
Z. Yusoff, J.H. Lee, W. Belardi, M. Ibsen, T.M. Monro and D.J. Richardson \\ Optoelectronics Research Centre, University of Southampton, Highfield, Southampton, SO17 1BJ, United Kingdom \\ Tel: +442380592483 Fax: +442380 593142,Email: jhl@orc.soton.ac.uk
}

\begin{abstract}
We demonstrate for the first time a Brillouin laser based on a Holey Fiber (HF). Using a simple Fabry-Perot resonator scheme containing a 75m long highly nonlinear HF with an effective area of $2.85 \mu \mathrm{m}^{2}$ we obtain a threshold of $125 \mathrm{~mW}$ and a slope efficiency of $\sim 70 \%$.

(C)2001 Optical Society of America

OCIS codes: (060.4370) Nonlinear optics, fibers, (140.3510) Lasers, fiber
\end{abstract}

The area of Holey Fiber (HF) technology [1] has progressed rapidly in recent years and has been successfully applied to the development of a number of nonlinear optical devices. For example, we recently demonstrated a nonlinear optical switch based on the Kerr effect in just 3.3m of HF [2], and a high gain Raman amplifier using just $75 \mathrm{~m}$ of fiber [3]. In this paper we show that HF technology can also be applied to another important class of nonlinear fiber-optic devices - namely those devices based on the Brillouin effect. More specifically, we provide what we believe to be the first experimental demonstration of a HF based Brillouin laser.

Our experimental setup is shown in Fig. 1. The pump source is based on an Erbium fiber DFB seed laser followed by a high power $\mathrm{Er} / \mathrm{Yb}$ Amplifier. The source has a linewidth of $\sim 35 \mathrm{kHz}$, a center wavelength of $1552.1 \mathrm{~nm}$ and provides a maximum output power of $\sim 750 \mathrm{~mW}$. The Fabry-Perot resonator incorporates a $73.5 \mathrm{~m}$ long holey fiber, a lens-coupled high-reflectivity cavity mirror and a $96 \%$ output coupler defined by the Fresnel reflection from the cleaved fiber facet at the pump launch end of the cavity. The pump beam was lens coupled onto one of the principal axes of the HF with a measured launch efficiency of $\sim 50 \%$. A 92:8 beam splitter was located at the pump launch end of the HF to monitor both the Brillouin laser output, and the incident pump power. A mechanical chopper was located in front of the HR mirror to facilitate alignment of the cavity. The holey fiber used in this experiment has a core diameter of $\sim 1.6 \mu \mathrm{m}$ and had a measured effective area of $2.85 \mu \mathrm{m}^{2}$ and a loss $40 \mathrm{~dB} / \mathrm{km}$ at $1550 \mathrm{~nm}$. The fiber is polarization maintaining by virtue of the structural hole asymmetry evident in Fig.1, and has a measured beat length of $\sim 0.4 \mathrm{~mm}$ around $1550 \mathrm{~nm}$. This fiber is more fully described in Ref. [3].

Fig. 2 shows experimental data of the Brillouin laser output power as a function of the input pump power. The laser threshold was found to be $125 \mathrm{~mW}$, and the slope efficiency $\sim 70 \%$. The RF spectrum of the beat signal between the laser output and the pump beam was measured using an RF spectrum analyzer, and confirmed that the frequency of the Brillouin laser output was downshifted by $10.6 \mathrm{GHz}$ relative to the pump frequency (see Fig. 3). We also investigated the stimulated Brillouin scattering (SBS) threshold within the HF. Feedback from the HR mirror was removed, and any possibility of Fresnel reflection from the terminated fiber at this end of the cavity was eliminated by immersing it in index matching liquid. We experimentally observed an SBS threshold of $\sim 280 \mathrm{~mW}$ (as shown in Fig. 2). This is in close agreement with our theoretical prediction of $240 \mathrm{~mW}$ based on our observation that the Brillouin linewidth is approximately an order of magnitude greater than that of pure silica. The origin of this broadening is tentatively thought to be due to structural variation along the fiber length.

In conclusion, we have experimentally demonstrated a $\mathrm{CW}$ Brillouin laser based on a highly nonlinear HF. A good laser power conversion efficiency of $\sim 70 \%$ and a Brillouin shifted output power of $110 \mathrm{~mW}$ were readily achieved at a wavelength of $1552.18 \mathrm{~nm}$ using a simple Fabry-Perot resonator scheme.

[1] J. C. Knight, T. A. Birks, P. St. J. Russell, and M. Atkin, “ All-silica single mode optical fiber with photonic crystal cladding,” Optics Lett., 21, 1547-1549 (1996)

[2] P. Petropoulos, T. M. Monro, W. belardi, K. Furusawa, J. H. Lee, and D. J. Richardson, "A 2R-regenrative all-optical switch based on a highly nonlinear holey fiber," Optics lett., 26, 1233-1235 (2001).

[3] J.H. Lee, Z. Yusoff, W. Belardi, T.M. Monro, P.C. Teh, and D.J. Richardson, "A holey fibre Raman amplifier and all-optical modulator," Proc. 27th European Conference on Optical Communication (ECOC’2001), PD.A.1.1, 46-47 (2001). 


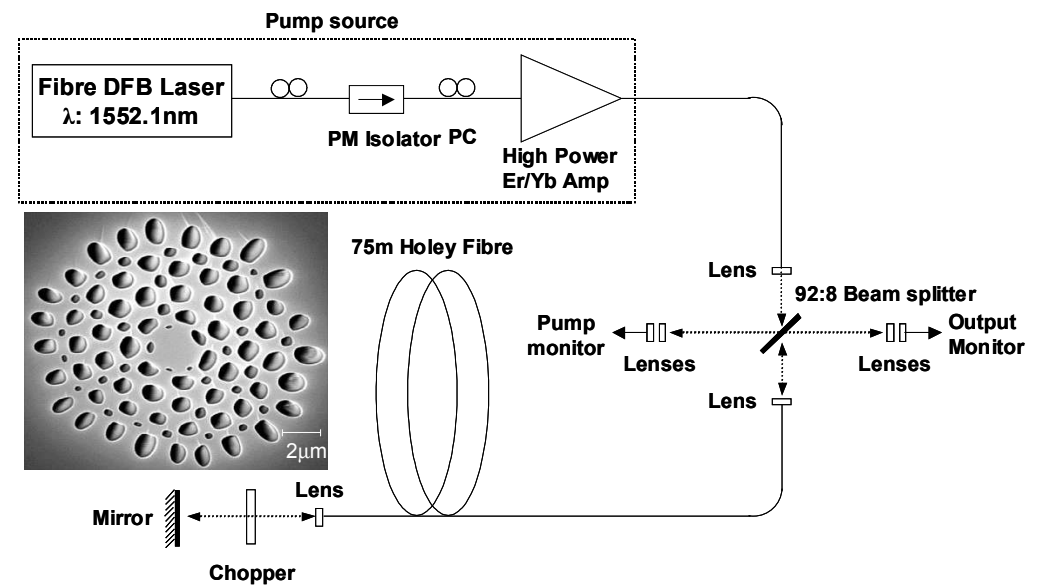

Fig.1. Experimental setup; inset: a cross-sectional SEM image of the HF used.

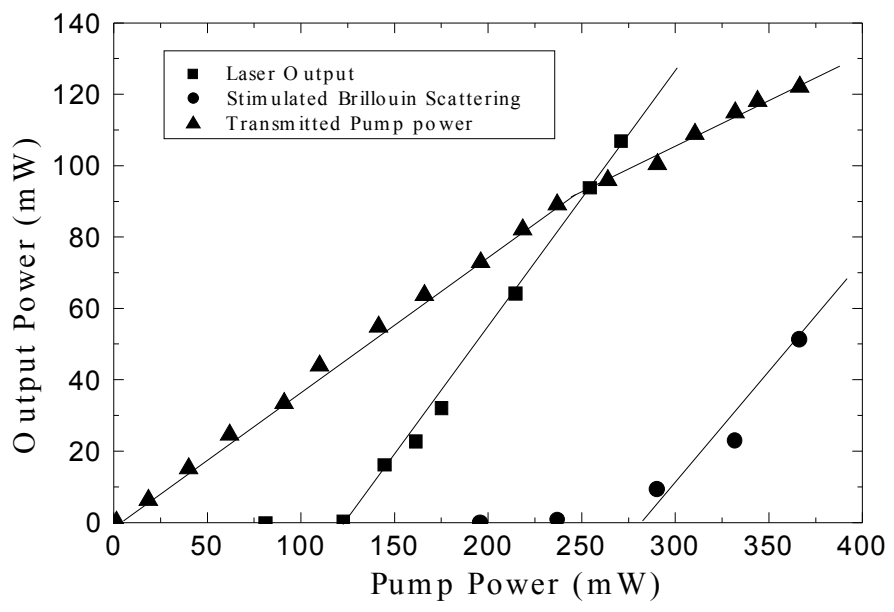

Fig.2. (i) Laser output, (ii) stimulated Brillouin scattering (backward), and (iii) transmitted pump power as a function of launched pump power (stimulated scattering only).

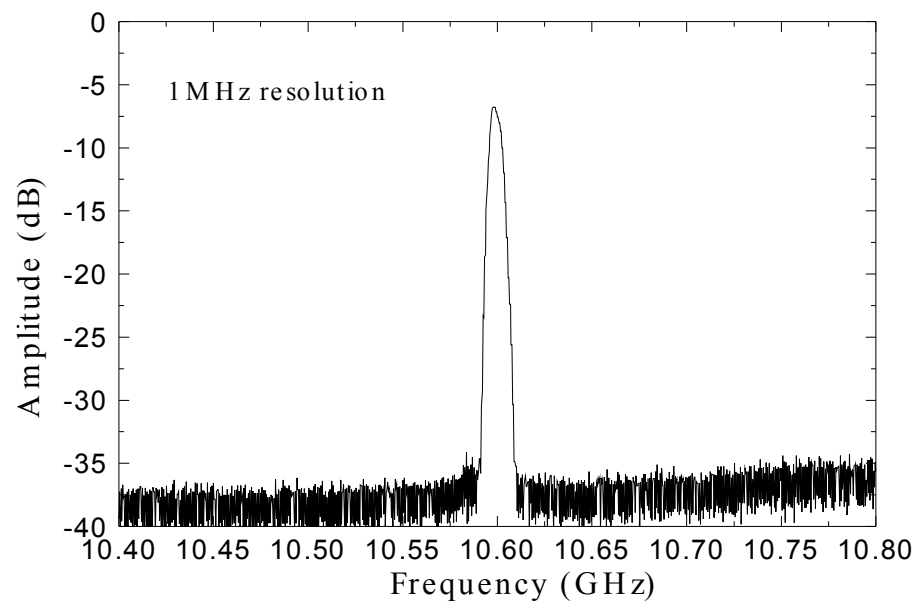

Fig.3. RF frequency trace of the beat signal between the Stokes and the pump beams. 